\title{
BMJ Open MAXimising Involvement in MUltiMorbidity (MAXIMUM) in primary care: protocol for an observation and interview study of patients, GPs and other care providers to identify ways of reducing patient safety failures
}

\author{
Gavin Daker-White, ${ }^{1}$ Rebecca Hays, ${ }^{1}$ Aneez Esmail, ${ }^{1}$ Brian Minor, ${ }^{1}$ Wendy Barlow, ${ }^{1}$ \\ Benjamin Brown, ${ }^{2}$ Thomas Blakeman, ${ }^{3}$ Peter Bower ${ }^{3}$
}

To cite: Daker-White $\mathrm{G}$, Hays R, Esmail A, et al. MAXimising Involvement in MUltiMorbidity (MAXIMUM) in primary care: protocol for an observation and interview study of patients, GPs and other care providers to identify ways of reducing patient safety failures. BMJ Open 2014:4:e005493. doi:10.1136/bmjopen-2014005493

- Prepublication history for this paper is available online. To view these files please visit the journal online (http://dx.doi.org/10.1136/ bmjopen-2014-005493)

Received 16 April 2014 Revised 24 July 2014 Accepted 28 July 2014

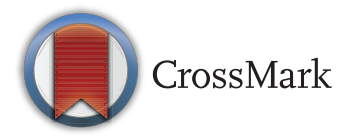

For numbered affiliations see end of article.

Correspondence to Dr Gavin Daker-White; gavin.daker-white@ manchester.ac.uk

\section{ABSTRACT}

Introduction: Increasing numbers of older people are living with multiple long-term health conditions but global healthcare systems and clinical guidelines have traditionally focused on the management of single conditions. Having two or more long-term conditions, or 'multimorbidity', is associated with a range of adverse consequences and poor outcomes and could put patients at increased risk of safety failures. Traditionally, most research into patient safety failures has explored hospital or inpatient settings. Much less is known about patient safety failures in primary care. Our core aims are to understand the mechanisms by which multimorbidity leads to safety failures, to explore the different ways in which patients and services respond (or fail to respond), and to identify opportunities for intervention.

Methods and analysis: We plan to undertake an applied ethnographic study of patients with multimorbidity. Patients' interactions and environments, relevant to their healthcare, will be studied through observations, diary methods and semistructured interviews. A framework, based on previous studies, will be used to organise the collection and analysis of field notes, observations and other qualitative data. This framework includes the domains: access breakdowns, communication breakdowns, continuity of care errors, relationship breakdowns and technical errors.

Ethics and dissemination: Ethical approval was received from the National Health Service Research Ethics Committee for Wales. An individual case study approach is likely to be most fruitful for exploring the mechanisms by which multimorbidity leads to safety failures. A longitudinal and multiperspective approach will allow for the constant comparison of patient, carer and healthcare worker expectations and experiences related to the provision, integration and management of complex care. This data will be used to explore ways of engaging patients and carers more in their own care using shared decision-making, patient empowerment or other relevant models.

\section{Strengths and limitations of this study}

The study will explore the role of multimorbidity in contributing to failures in access, communication or relationship and management continuity in primary healthcare.

- Findings will be used to design candidate interventions with the aim of encouraging multimorbid patients and their informal carers to become more actively involved in condition management.

- A small number of vulnerable cases will be purposefully sampled with the aim of highlighting potential precursors to safety failures, although this design means that findings will not be generalisable to all patients with multimorbidity.

\section{INTRODUCTION}

Patient safety has been defined as the 'avoidance, prevention, and amelioration of adverse outcomes or injuries stemming from the processes of healthcare'. ${ }^{1}$ To date, most research on patient safety has focused on hospital settings where safety incidents can include surgical errors, such as a patient receiving the wrong operation. ${ }^{1}$ As the care provided within primary care is less technical than that provided within hospitals, there could be a perception that safety is not a problem in this setting. However, $85 \%$ of contacts with the National Health Service (NHS) take place in primary care. In the $\mathrm{UK}$, there are 300 million general practice appointments and over 900 million prescription items dispensed each year. This means that nearly 750000 patients consult their general practitioner (GP) each day and 1.6 million people visit a pharmacy daily (1.2 million for health reasons). ${ }^{2}$ Therefore, the potential for safety incidents is huge, but little is known about patient safety in this 
context. A review of studies of the frequency of errors in primary care suggested that there are approximately 580 safety incidents per 100000 consultations, which in the UK would translate to around 4350 incidents per day. ${ }^{2}$

Multimorbidity, defined broadly as the coexistence of two or more long-term health conditions, is an increasingly 'normal' experience for people in later life and presents challenges for patients in respect of continuity of care, ${ }^{3}{ }^{4}$ care planning ${ }^{5}$ and self-management of conditions. ${ }^{6}$ Patients with multimorbidity also face problems associated with polypharmacy, ${ }^{7}$ and patients and professionals need to weigh the anticipated benefits of additional treatments against potential harms and burdens. ${ }^{8}$ Polypharmacy and repeated referrals for specialist care have the potential to increase the risk of adverse clinical events, including adverse drug events. ${ }^{9}$ An Austrian study of polypharmacy in general practice found that rates of medication errors were high and the authors recommended that older patients with multimorbidity would benefit from external medication reviews. ${ }^{10}$ However, a qualitative study of UK-based pharmacists found that some felt that patients with complex conditions-which include proportionately more patients with multimorbidity-lie outside of their existing skill set. ${ }^{11}$ In addition to the specific issue of polypharmacy, patients with multimorbidity also face the possibility of receiving contradictory health advice from health professionals (consulted about single conditions, perhaps) and might face difficult self-management options in that context. Thus, for a variety of reasons, elderly patients with multimorbidity are likely to be at increased risk of safety failures when compared with other patient groups. Furthermore, some groups of multimorbid patients may face additional vulnerabilities in relation to patient safety issues. These might include patients with low health literacy, physical frailty, anxiety or depression and/or low-socioeconomic status.

\section{Previous patient safety research}

Traditionally, patient safety research and initiatives have focused on medical error, and conceived of errors of 'execution,' as distinct from errors of 'planning' in treatment modalities. ${ }^{12}$ Most of this research has been conducted in hospital settings where many adverse events are related to surgery. However, it is questionable whether these findings could be translated to primary care settings. ${ }^{13}$ 'Machine' or systems thinking predominates in hospital-based studies of patient safety, which have tended to point to restructuring care organisation in order to reduce clinical uncertainties and medical errors. ${ }^{14}$ However, everyday clinical work in general practice is laden with uncertainties, and treatment and diagnosis can often involve a 'let's wait and see' approach. ${ }^{14}$ Furthermore, while patient safety strategies have been focused towards the knowledge and actions of frontline clinical staff, patients and their informal carers also contribute towards systems of patient safety, which points to the need for a collaborative approach. ${ }^{15}$
Ethnographic approaches have been widely employed in hospital-based studies of patient safety, including those funded through the national Patient Safety Research Programme (PSRP) in the UK. A narrative review of four such studies, by Dixon-Woods, found that all research teams had been challenged by the definition and classification of 'patient safety incidents' and each adopted a different approach. Thus, the author argued that 'ethnographic work in patient safety is always likely to be engaged in normative judgements about how things ought to be, as well as describing how things are' (latter emphasis added). ${ }^{16}$

These ethnographic studies also varied widely in relation to the subjects and objects of observation or interaction. McDonald et $a l \mathrm{~s}^{17}$ study of patient safety in an operating theatre rested on an approach that involved researching the 'safety culture' of the organisation, by observing individual and group beliefs, perceptions, competencies and patterns of behaviour. The Healey and Vincent study explicitly compared individual and team factors in the operating theatre and included a 'task-specific operational assessment' to assess safety issues. ${ }^{18}$ The Catchpole et al operating theatre study similarly adopted a systems perspective and also used expert observers and video capture, and assessed the 'cognitive and social abilities' of staff using a scale adapted from the aviation industry. This study would be better described as mixed-methods rather than ethnographic, as it included the mathematical modelling of systems conditions on patient safety. ${ }^{19}$ Finally, in the English A\&E study cited by Dixon-Woods, ${ }^{16}$ observational methods were part of a broader mixed-methods study programme, and were focused on staff communication and triage processes. Owing to the case-mix and variability in care pathways in A\&E departments, this study, 'concentrated on looking at safety aspects of processes that aid clinical decision-making rather than the care of clinical entities themselves'. ${ }^{20}$

Other ethnographic studies of patient safety include a US hospital-based study of adverse events that was based on observation of routine clinical meetings and focused on events identified and raised by staff members themselves. These events were categorised into nine problem areas by the researchers: diagnosis, surgery, treatment, monitoring and daily care, drugs/medication, nutrition, anaesthesia, complications and 'other'. ${ }^{21}$

It can be seen that while previous ethnographic studies of hospital safety have adopted a variety of methods and perspectives, the focus has tended to be on clinical systems or staff knowledge or behaviours. This approach is perhaps best exemplified by an ethnographic study of patient safety in relation to intravenous interventions in UK hospital settings. ${ }^{22}$ Here, observation by clinically trained researchers focused on the preparation and administration of intravenous drugs by healthcare workers. In a small number of cases, the researchers intervened to prevent the patient from receiving the preparation due to errors. ${ }^{22}$ In addition, 
there has been a reticence, particularly in the context of the US private health system, for research studies to systematically collect linked case data from patients and carers due to concerns about potential medical malpractice litigation. ${ }^{12}$

Given that findings of hospital-based patient safety studies are not generally translatable to primary or ambulatory care settings, several research projects have been undertaken to define the most important patient safety issues in primary care. These studies have sought to garner the views of doctors, patients or both. A US population-based qualitative study of 38 adults living in Virginia and Ohio set out to solicit stories of "preventable problems with primary healthcare that led to physical or psychological harm'. ${ }^{23}$ In the UK, a qualitative study of 33 patients living with long-term conditions similarly set out to characterise patients' perspectives of what constitutes 'error' in primary care. ${ }^{24}$ A New Zealand-based study used a nominal group technique to examine patients' and professionals' views of patients' contribution to error. ${ }^{25}$ The salient findings from these three studies are presented in table 1. In contrast to hospital-based studies, access, communication and relationship issues appeared more prominent than technical medical errors. ${ }^{23}$ Transition points between primary and secondary care were highlighted as sites where there is an increased risk of errors, or circumstances that may lead to errors later in the care pathway. ${ }^{24}$ We will develop this taxonomy of events further in order to frame the collection and analysis of data for the study. This will be achieved via a metasynthesis of qualitative studies of patient safety in primary care (work in progress).

\section{Methodologically relevant previous studies}

The methods of our study are informed by other closely related ethnographic studies in primary care that did not have an explicit focus on patient safety. Most relevant of these is a UK-based ethnographic and serial interview study of the coordination of care for people in the last year of life living with a progressive condition. ${ }^{26}$ In this study, observation and interviews were conducted in three different health settings: primary care, outpatients and an acute setting. As in other studies, researchers gained access to the field through shadowing staff, rather than patients. The primary data collection method for linked patients and family carers consisted of semistructured interviews undertaken every 8-12 weeks for a period of 5-9 months (or until death). According to this schedule, patients were seemingly interviewed between two and three times during the $<9$ month course of the study. We discuss the distinctions between an ethnographic approach and a 'longitudinal qualitative' approach in the Methods and Design section below.

\section{A framework for the study}

As was noted above, the findings of hospital-based studies of patient safety do not appear to translate fully into primary care. However, they do seem to inform the technical, management and coordination aspects of the

Table 1 Taxonomy of perceived events that could lead to subjective harm in primary care, as found in three qualitative studies $^{23-25}$

\begin{tabular}{|c|c|c|}
\hline Domain & Patient contribution & Staff/system contribution \\
\hline Access breakdown ${ }^{23}$ & $\begin{array}{l}\text { Untimely attendance } 25 \\
\text { Underattendance } 25 \\
\text { Culture given priority over health } \\
\text { needs }^{25}\end{array}$ & $\begin{array}{l}\text { Telephone access }^{23} \\
\text { Gatekeeping }{ }^{2324} \\
\text { 'In system access' } \\
\text { Access to specialist care } 2324\end{array}$ \\
\hline Communication breakdown ${ }^{23}$ & $\begin{array}{l}\text { Inarticulateness } \\
\text { Low literacy }^{25} \\
\text { Taciturnity }^{25} \\
\text { Comprehension errors } \\
\text { Lack of confidence }^{25} \\
\text { Artfulness } \\
\text { Memory errors } \\
\text { Me }\end{array}$ & $\begin{array}{l}\text { Improper appointment scheduling } \\
\text { Failure to respond to reports of adverse drug reactions } \\
\text { or painful symptoms }\end{array}$ \\
\hline $\begin{array}{l}\text { Errors of coordination/management } \\
\text { continuity }{ }^{24}\end{array}$ & $\begin{array}{l}\text { Comprehension errors } \\
\text { Memory errors } \\
\text { Knowledge errors } \\
\text { Kn }^{25}\end{array}$ & $\begin{array}{l}\text { Administrative errors in recording, posting, updating } \\
\text { Transfer of records to and from practice }{ }^{23} 24 \\
\text { Wrong chart used for patient }{ }^{23}\end{array}$ \\
\hline Relationship breakdown ${ }^{23}$ & $\begin{array}{l}\text { Selfishness } \\
\text { Perception of not being taken } \\
\text { seriously }^{24}\end{array}$ & $\begin{array}{l}\text { Inadequate time with clinician } \\
\text { Intermediary necessary } \\
\text { Care not by usual clinician } \\
\text { Disrespect or insensitivity }\end{array}$ \\
\hline Technical errors $^{23}$ & $\begin{array}{l}\text { Low literacy } \\
\text { Sharing of medication } \\
25 \\
\text { Duration errors in medicine } \\
\text { taking }^{25} \\
\text { Errors in self-management }{ }^{25} \\
\text { Failure to take treatment }\end{array}$ & $\begin{array}{l}\text { Incomplete medical history } \\
\text { Deficient/incomplete examination or investigation } \\
\text { Wrong, incomplete or delayed diagnosis } \\
\text { Wrescribing errors } \\
\text { Pre } \\
\text { Inadequate patient education re: diagnosis or } \\
\text { treatment }^{23}\end{array}$ \\
\hline
\end{tabular}


framework in table 1. By using a framework of literature concerning patient perspectives of safety issues in primary care, we are explicitly basing our own study on previous work concerning patients' views. This has advantages for ethical as well as methodological reasons. In particular, it means that our work is built on previous knowledge and findings, which is not always the case in qualitative studies, where research is sometimes replicated in saturated fields, perhaps without reference to the extant literature. ${ }^{27}$

In order to improve quality of care for older adults with multimorbidity, a tailored approach involving patientcentred goals has been advocated, ${ }^{28}$ which also fits with current expert guidance for clinicians. ${ }^{8}$ Given that patients and informal carers are already involved in 'safety work' (such as checking or documenting medications or questioning staff), some studies have examined the potential for patients to become more engaged or involved. A literature review identified five groups of factors that can affect patients' involvement in patient safety: patient-related, illness-related, healthcare professional-related, healthcare setting-related and task-related. ${ }^{29}$ Patients with multimorbidity tend to be older and disproportionately represented in areas of socioeconomic deprivation. ${ }^{30}$ Such patientrelated factors are likely to affect knowledge, beliefs and engagement. For example, the literature review found that older patients are less likely to want to be more involved in their care. ${ }^{29}$ From the material presented thus far in this protocol, it is also clear that patients with multimorbidity have a greater illness burden (illness-related factors) as well as presenting clinical and organisational challenges for healthcare professionals and healthcare delivery. The authors of the review conclude that "patients should only be seen as safety safeguards when they want and are able to. ${ }^{29}$

In a different review, the same authors examined the opportunities for 'active patients' to improve patient safety, although this was in the specific context of a surgical care pathway. ${ }^{31}$ However, the article is most useful for underlining that patients require knowledge to participate as well as an ability and willingness to do so. The attention to knowledge in the 'active patient' model is of particular relevance to our own study, especially given the focus on relationship and communication issues found in the literature review (work in progress):

A major barrier to patient empowerment and, in turn, patient involvement is professional defensiveness and resistance to move away from the paternalistic viewpoint that "physician knows best.... Preliminary evidence suggests that physicians may be culturally averse to increasing patient involvement because of the negative psychological impact that discussion of risk may have to the patient. [31: page 42]

This brings us back to our earlier observation that the 'normal' uncertainties which characterise clinical work in general practice are likely to make this process much more challenging for patients and doctors alike.

\section{Research objectives}

Our core aims are to understand the mechanisms by which multimorbidity leads to safety failures, to explore the different ways in which patients and services respond (or fail to respond), and to identify opportunities for intervention through a variety of platforms, such as enhanced care planning, monitoring and feedback of patient experience and through intervention at the patient and organisational level.

The relationship and communication domains in table 1 highlight the potential role that patients might play in patient safety themselves. The patient safety literature underlines that promoting safe care is a collaborative enterprise in which patients are already involved, ${ }^{15}$ although we assert that this is likely to be more challenging for patients with multiple health problems (see above). Thus, the ultimate objective of our study is to explore and examine ways in which patients (or their informal carers) might become more actively engaged (or better involved) in their own care, with the aim of reducing the potential for safety failures. Hence, we have called our study 'MAXIMUM': MAXimising Involvement in MUltiMorbidity (MAXIMUM) in primary care.

\section{Patient involvement (research user group)}

Public and patient involvement lies at the core of our study, given our focus on patients and carers as agents in primary care patient safety. We will follow the principles of Boote $^{32}$, who is also undertaking an independent evaluation of the whole of the Greater Manchester Patient Safety Translational Research Centre's (PSTRC) research portfolio, in terms of consumer involvement. The management of our study includes input from a 'Research User Group' (RUG) of patient volunteers who offer advice on the study process and outcomes from their own perspective. Our RUG members (authors BM and WB) were involved in the design of this protocol-specifically in relation to ethical issues around research burden (see below) -and will also offer their own unique perspective during the data collection and analysis phases. Their insights will be particularly valuable when we come to develop and evaluate potential candidate interventions to improve patient and carer involvement.

\section{METHODS AND ANALYSIS \\ Overview}

The study draws on two methodological strands in qualitative research: ethnography ${ }^{33}$ and 'longitudinal qualitative ${ }^{34} 35$ or 'serial' interview studies. ${ }^{36}$ The approach is best exemplified in a series of articles outlining a programme of research concerning the coordination of care for individuals with advanced, progressive conditions near the end of life. ${ }^{26} 36{ }^{37}$ However, we will aim to privilege observation methods over interviews, given that 
communication and interaction are of utmost importance in primary care. Ethnography is traditionally the method of social anthropologists, who would spend prolonged periods of time in participant-observer roles in alien or marginal cultures in order to understand cultural signs, meanings and practices. However, it is also common for ethnographic research projects to be focused and undertaken with applied intent in health services research contexts. Finally, the study also draws on case study methods, for example, as in a Nursing study of life course adjustment to multimorbidity. ${ }^{38}$

\section{Setting}

This study will take place within primary care practices across four different districts within Greater Manchester. Greater Manchester is an economically diverse region with measures of social deprivation and health outcomes that rank among the worst in the country. ${ }^{39}$

\section{Participants}

The sampling strategy is designed to maximise the richness of data while minimising the potential research burden for patients and general practices.

GPs: General practices will be identified via Clinical Commissioning Groups. Eight different practices will be purposefully selected to achieve variation in patient list size, number of GP partners/locums, and availability of different services within the practices. We will seek to recruit one GP from each practice (thus, 8 in total). This will minimise the research burden on general practices as well as allowing us to 'control' for issues particular to the GP providing care in each case. Each GP will be asked to help us recruit 3-5 of their patients (see below).

Patients: People with multimorbidity, considered to be at increased risk of safety failures, will be recruited. All patients will have more than two active health conditions, but we will aim to recruit people with larger numbers of conditions who are potentially vulnerable to safety failures in other ways, such as:

- They have a large number of health appointments each year.

- They are prescribed a large number of medications.

- They are at an increased risk of falls (eg, because they have arthritis or suffer from a neurodegenerative condition).

- They have communication difficulties or low health literacy (whether because of language, learning disabilities or dementia).

- They live alone.

- They also have anxiety or depression.

These groupings are neither mutually exclusive, nor exhaustive, although they demonstrate how we will purposefully sample groups and individuals deemed to be at increased risk of patient safety failures.

The study may include patients with cognitive, speech or other deficits that affect communication. Both researchers working on the study have prior experience of qualitative interviews of elderly people with health problems. One researcher also has experience of interviewing patients with disordered speech due to neurological problems. Both have experience of interviewing non-English speaking patients through family, carer or professional interpreters. We will not exclude patients on the basis that they do not speak English. If GPs identify a non-English speaker, we will employ an interpreter to work specifically with that patient. We will check with the GP that the use of an interpreter is appropriate and not likely to lead to excessive burden on any non-English speaking patients, who may already have an interpreter present for their clinical care.

\section{Sample size: patients}

We will initially seek to recruit a minimum of three patients through each participating GP. Depending on the quality and quantity of data we are able to collect (subject to service use and consent), we may seek to recruit more patients (to a maximum of five per practice/participating GP, or a total sample of 40 patients).

The number of participants has been chosen to allow for comparisons between smaller, subgroups of patients (eg, those with different vulnerabilities). Patients will also be asked to identify three other individuals involved in their care (see below).

\section{Sample size: other care providers}

As outlined below, we will also seek to recruit additional people involved in the care of the participating patients. Approximately three additional healthcare workers or paid or informal carers will be recruited per patient.

\section{Study procedures: recruitment}

GPS

Potential GP participants will be sent a letter of invitation, which will be followed up with a telephone call. Further information will be provided in a visit to the practice, and written informed consent will be sought. Recruitment began in March 2014, with the first GP being recruited in June.

\section{Patients}

Patient participants will be recruited from the patient lists of each participating practice. GPs will identify potential participants and contact the patient (by letter or telephone) to provide initial information on the study. Interested patients will be asked to pass on their contact details to the research team (either directly or through their GP). A member of the research team will then contact the potential patient participant to answer any initial questions they may have and arrange to visit them (at a convenient time) to discuss the study in detail, and seek written informed consent. Where appropriate, we will then conduct a short interview with the patient to ascertain their perceptions or knowledge of their main symptoms, health problems and active treatments. We will also ask the patient to identify who is 
currently involved in their care, including health and social care workers, informal carers and family members. At the time of writing the final version of this manuscript (July 2014) the first patients were in the process of being recruited.

\section{Other care providers}

Recruitment procedures for other care providers will be developed in consultation with the patient participants. Possible methods include: providing the patient participants with information sheets to pass on to their care providers, and asking these individuals to contact the research team to arrange a visit to discuss the study; or, a member of the research team discussing the study with a care provider who is present during a visit to the patient's home.

\section{Data collection}

Data will be collected longitudinally over 2 years, from August 2014 to December 2016.

\section{Extraction of data from the primary care medical record}

Data will be extracted from the patients' medical records only for the period during which participants are included in the study (ie, we will not be looking back historically through the record). Data will be extracted concerning current appointments, prescriptions and health conditions. The data will be anonymised at the point of extraction using a participant ID code rather than the participants' personal data (such as their name, address or NHS number). The data will be transported on an encrypted laptop or other encrypted portable device, then uploaded onto a secure University file server and securely deleted from the encrypted device.

This material will be extracted every 12 months (ie, at 0,12 and 24 months). It will be compared with and used to support the data provided by the patient (see above). The collected data will also be reviewed by the clinical members of the study team so they can inform the fieldworkers of the medical and service delivery issues for each patient, so that they have some context and are aware of the likely staff who will be involved and the clinical procedures and guidelines that might be relevant to the patient. We will not be 'objectively' reviewing the medical record data, for example, to find prescription errors. However, we will examine the extent to which the information (eg, on the number or nature of conditions or diagnoses) chimes with doctors' narratives around each case or patients' accounts of their own health problems or illness management.

\section{Observation of patients' interactions or consultations with} health professionals, care workers or informal carers

We will seek to observe two consultations per year between each participating patient and their GP, and one interaction per year between each participating patient and three of their other care providers; that is, a total of five observations per participating patient per year. This figure is based on methodological guidance related to a similar longitudinal qualitative study. ${ }^{28}$ If the maximum number of 40 patients are recruited to the study, this would lead to a data set consisting of 400 patient interactions with health workers or carers over the 2-year course of the study (ie, 20 per participating GP, 10 per participating patient and 2 per participating other care provider). Following the same guidance, the research team will not set specific time points for these observations to take place but we will flexibly respond to patient care episodes and be guided by a timetable that is most convenient for the participants. ${ }^{37}$ The consent provided for observation and, where appropriate, for audio-recordings of the consultations or interactions to be made, will be checked with all participants immediately prior to the observation. Where audio-recordings are made, consent will be recorded verbally at the start of the recording.

Flexible, short interviews with patient participants about interactions or consultations (observed or not observed)

Following each observed interaction or consultation, participating patients will be invited to participate in a short interview where they will be asked about their opinions or reflections on the interaction. These interviews could take place in the NHS primary care setting where the observation occurred (if a private room can be found), patients' own homes or by telephone. If it is not possible to interview participating patients after each observed interaction or consultation, interviews could be arranged following interactions or consultations that have not been observed. As above, consent provided for interviews and, where appropriate, for audio-recordings of the interviews to be made, will be checked immediately prior to the interview.

\section{Interviews with patients, health professionals, care workers or informal carers}

To obtain more in-depth information, a small number of semistructured interviews will also take place with all participants.

We will seek to interview participating patients annually about general aspects of care ('How are things going for you?'). We will also interview participating GPs annually about each of their participating patients' conditions, management and care plan. We may discuss multiple patient participants during the same interview or in separate interviews according to the preferences of the GPs. We will also seek to interview a subsample of the other participating care providers about their perceptions of the care experience. This subsample will consist of those individuals who the patient participants identify as being particularly important to them. We expect this sample will primarily consist of informal carers or health workers in specialist clinics (eg, diabetes clinics) or personal (home) care. If the maximum number of 40 patients are recruited to the study, this would lead to a data set consisting of 80 patient and 80 
GP interviews (2 of each or 4 in total per patient). Assuming that one other participating care provider per patient (who is considered particularly important) is also interviewed at some stage during the life of the study, that would lead to a total of five patient or GP or health carer semistructured interviews per patient, to add to the 10 observations of interaction episodes and 10 brief postconsultation interviews, as detailed above.

Given the ethnographic nature of the study, the content of patient, GP and carer interviews will be derived iteratively from the early stages of the fieldwork and data collected. However, and as described above, an overall framework for these discussions has been derived from the existing literature (table 1). Thus, our initial topic guide for GPs focuses on their perception of participating patients' different conditions and needs; how these are managed in clinical practice; and, their perception of the quality of the doctor-patient relationship and any communication issues. Their perceptions about the quality of care provided by others and any personal or characteristics of each patient's home environment or personal circumstances that might be seen to impact on the care plan. The starting point for the patient topic guide focuses on their perceptions and experiences of the care they receive from GPs, other health professionals and any informal carers. Again, issues in communication and relationships will be explored so that they may be compared with the opinions derived from health professionals and informal carers. Another main focus of the patient interviews will be on the work involved in managing or self-managing their conditions, such as getting to health appointments, obtaining or taking medications or other treatments or therapies.

\section{Diary data from patients about aspects of care}

At study recruitment, we will explore with participants if (and under what circumstances) they would like to provide additional information about their care and their interactions with health professionals, care workers or informal carers. This is an 'optional' component of the study. This data will be especially useful in cases where we are unable to observe a reasonable proportion of the interactions a patient has with carers or health workers, either because consent is not provided or because the patient has a very high number of health appointments or interactions (eg, if they see their GP once a month or more). At recruitment, we will ask participants how they would like to provide such information. Options will include by written or recorded diary (via voice or video on personal tablet computers), or by telephone or email (instigated either by the participant or the research team). Equipment or a contribution to the cost of patients' telephone or internet bills will be provided by the research team as appropriate.

\section{Data analysis}

This is an applied ethnographic study that involves both inductive and deductive analytic techniques.
Anonymised field notes, transcripts of interviews and observations, and diary data will be analysed with the aid of NVIVO (computer software designed for the management of qualitative data). Analysis will involve comparing data for individual patient participants and subgroups of participants (eg, those with different vulnerabilities) with one another, and identifying themes. We will also look at how care is managed in participants' own homes, and in different primary care settings. This analysis will be carried out using a framework developed from existing literature on patient safety in primary care (table 1 and meta-synthesis work in progress). This will include themes such as: access to services, communication and relationship factors, technical errors and the coordination or continuity of care. The analysis will focus on potential precursors to patient safety failures and, where patient safety and quality of care could be improved.

\section{ETHICS}

The study presents a range of ethical issues, which are considered below.

\section{Research burden}

The aim of an applied ethnographic study of primary and community-based care necessarily involves getting as close as possible to research participants in order to observe how they deal with symptoms, medicines, health workers and informal carers on a daily basis. However, such a research approach has the capacity to cause additional burdens to a group of people selected precisely because they already have 'a lot on their plate.' The principal means by which we will limit research burdens for patients and general practices is by only recruiting small numbers of patients from each practice; by adopting a mainly observational style of data collection; and by careful consideration of the number and frequency of research procedures. Furthermore, we will be flexible in the ways that we collect data on ongoing health service use; tailoring our approach to the particular circumstances of different patients. However, the patient representatives on our study team have suggested that research burden will not be an issue for participants who will in fact enjoy the opportunity to discuss their perceptions of the care they receive.

\section{Confidentiality and data protection}

Data will be collected and stored according to current research governance frameworks. Only those researchers who are actively involved in collecting research data will routinely have access to identifiable participant information (eg, demographic and contact details). Electronic data will be encrypted and stored on a secure file server with restricted access, and paper documents will be held in a locked cabinet within a locked room. These data will also be securely destroyed when no longer required. For data manipulation, storage and analysis, participants 
will be given a unique identification number or pseudonym.

Given that this study concerns patient safety, it is possible that some potential participant GPs and other care providers could feel they would be scrutinised through our observations. This may lead to recruitment problems. We will ensure that all participants understand that the aim of the research is not to evaluate the care that is provided, but to identify the ways in which multimorbidity can lead to safety problems. We will also stress that all research data will be anonymised, and that participants' identities will be protected.

\section{Clinical issues}

The fieldworkers (RH and GD-W) working on this study are not clinically trained, and are thus unlikely to identify technical safety issues (eg, those that concern the dose or types of medication a patient has been prescribed). At the end of this study, all participants will be provided with a summary of our findings. This will include a list of the potential precursors to patient safety failures that we identify, which will enable both patients and care providers to reflect on their own practice and behaviour. If we identify a serious risk to the safety of a patient participant or someone connected to them, during the course of the study, this will be reported to the patient's GP. We will inform all potential patient participants that we will do this, and they will be required to agree to this as part of the informed consent process.

In deciding what to report, we will use an 'escalator' system. The fieldworkers will initially discuss any concerns with a clinical member of the Greater Manchester PSTRC (BB, TB or AE), where the study is based. In all cases where we have any serious concerns, these will be communicated to patients' own GPs by the senior clinical member of the research team (AE).

The fieldworkers will not routinely assess the mental state of participating patients. However, if a fieldworker encounters a patient who articulates a desire or plan to harm themselves, we will use a formal risk assessment tool and follow the specified procedure. The outcomes of this formal assessment might involve no action, recommending the patient speaks to their GP or contacting the emergency services.

\section{Consent and loss of capacity to consent}

People unable to give informed consent will be excluded from the study. The assessment on capability of potential participants will be made by GPs. Initial consent will be in writing. Further ongoing consent ${ }^{35}$ will be obtained orally and recorded where appropriate (eg, in the context of a follow-up telephone interview). Our sample will include frail, vulnerable and mainly elderly people. Some may have early signs of dementia or have a mental illness. In such cases, even where we consider that individuals are capable of giving informed consent, we will be attentive to the advice of carers and will withdraw from the field when we believe that it is in the best interests of a patient.

If a patient participant loses capacity to consent during the study, we will seek to identify a consultee who can advise the research team about the patient's ongoing participation in the study, in line with statutory legislation. Where no consultee can be identified, the participant will be withdrawn.

\section{Researcher/subject issues}

Longitudinal qualitative studies place increased demands on researchers who face a variety of dangers, for example, 'burn out'. The fieldworkers, who are experienced qualitative researchers, will hold field work debrief sessions once a week during data collection. A professional occupational counselling service is available at the researchers' place of work, if needed. From the point of view of the research participants, there is the potential for a minority to become somehow dependent on the researcher (eg, as a source of help and support when they go to see their doctor or other health workers). ${ }^{35}$ Termination of the data collection phase will have to be handled sensitively. If necessary, the fieldworkers will continue to occasionally visit study participants for the full duration of the study (and not just the 'data collection' phase).

\section{DISCUSSION}

\section{Strengths and weaknesses}

A longitudinal consideration of interaction and clinical management in patients with multimorbidity in primary care has the potential to explore the circumstances in which relationships or significant events might act as precursors to potential errors or adverse events. The management of multimorbidity presents significant challenges for patients, doctors and carers and is likely to exacerbate issues in communication, relationships or systems that have the potential to bring errors or adverse events in medical care. A qualitative approach will allow us to explore cases in depth and detail and incorporate the views and perceptions of patients, doctors and significant others involved in care. The design of the study means that findings will not be generalisable to all patients with multimorbidity in primary care. However, such an approach has the capacity to yield authentic accounts which can be considered 'trustworthy' or valid in a conceptual sense. These real world accounts will be used to inform the development of interventions targeted mainly at patients or their informal carers with the aim of improving the quality of patient care. The study population includes patients with additional vulnerabilities, where relationship, communication and issues in continuity of care are likely to be magnified. While this is necessary in order to increase the likelihood of identifying precursors to patient safety failures, it means that our findings may not be relevant for other patients who 
are better equipped to be actively engaged in their own care.

\section{Conclusion}

Patients with multiple long-term conditions are likely to face an increased risk of safety failures in primary care. This study adopts an applied ethnographic approach in order to explore issues, incidents and trajectories that could be seen to lead to deficiencies or failures in treatment management or care. Although our study is qualitative and exploratory in nature, we will adopt a highly focused approach based on the existing literature concerning patient safety in primary care. We will follow a 'cohort' of cases over time. Given the need for 'tailored' approaches in the clinical management of multimorbidity (in contrast to the guidelines-based approach ie, usual in single conditions), ${ }^{29}$ an individualised 'case' approach is likely to be most fruitful for exploring the mechanisms by which multimorbidity leads to safety failures.

Following from the literature, we conceive that an important way to reduce the possibility of errors or failures in care is by better engaging and involving patients and their informal carers in 'patient safety culture.' However, the contexts of ageing and multimorbidity present a range of challenges in this respect, such that some interventions are likely to be more useful or important than others. Our study follows in the tradition of other ethnographic and qualitative studies of patient safety, although we will adopt an approach firmly grounded in patient perspectives and experiences.

The study presents a range of ethical challenges. In considering these, we have taken advice from researchers working on related studies and the Research User Group members of the study team (BM and WB). We have also developed an 'escalator' system to alert the relevant authorities, should any serious risks to safety be identified.

\section{Author affiliations}

${ }^{1}$ NIHR Greater Manchester Primary Care Patient Safety Translational Research Centre (Greater Manchester PSTRC), University of Manchester, Manchester, UK

${ }^{2}$ Centre for Health Informatics, Institute of Population Health, University of Manchester, Manchester, UK

${ }^{3}$ Centre for Primary Care, Institute of Population Health, University of Manchester, Manchester, UK

Contributors PB conceived the study. PB, GD-W, RH, AE, BB and TB were all involved in the design of the study. BM and WB provided lay advice on the design of the study from a patient's perspective. The manuscript was drafted by GD-W and RH. All authors reviewed and approved the final version of the manuscript.

Funding This study is funded by the National Institute for Health Research Greater Manchester Primary Care Patient Safety Translational Research Centre (NIHR GM PSTRC). The views expressed are those of the author(s) and not necessarily those of the NHS, the NIHR or the Department of Health.

Competing interests None.

Ethics approval NHS Research Ethics Committee (REC) for Wales (Ref: 14/ WA/0037, 13/02/2014)

Provenance and peer review Not commissioned; externally peer reviewed.
Open Access This is an Open Access article distributed in accordance with the Creative Commons Attribution Non Commercial (CC BY-NC 4.0) license, which permits others to distribute, remix, adapt, build upon this work noncommercially, and license their derivative works on different terms, provided the original work is properly cited and the use is non-commercial. See: http:// creativecommons.org/licenses/by-nc/4.0/

\section{REFERENCES}

1. Vincent C. Patient safety. London: Churchill Livingstone Elsevier, 2006.

2. Sanders J, Esmail A. The frequency and nature of medical error in primary care: understanding the diversity across studies. Fam Pract 2003;20:231-6.

3. Haggerty JL. Ordering the chaos for patients with multimorbidity: building continuity of care takes work but earns trust [Editorial]. BMJ 2012;345:e5915.

4. Cowie L, Morgan M, White $\mathrm{P}$, et al. Experience of continuity of care of patients with multiple long-term conditions in England. $J$ Health Serv Res Policy 2009;14:82-7.

5. Newbould J, Burt J, Bower $P$, et al. Experiences of care planning in England: interviews with patients with long term conditions. BMC Fam Pract 2012;13:71.

6. Bower P, Hann M, Rick J, et al. Multimorbidity and delivery of care for long-term conditions in the English National Health Service: baseline data from a cohort study. J Health Serv Res Policy 2013;18 (2 Suppl):29-37.

7. Hunt LM, Kreiner M, Brody $\mathrm{H}$. The changing face of chronic illness management in primary care: a qualitative study of underlying influences and unintended outcomes. Ann Fam Med 2012;10:452-60.

8. American Geriatrics Society Expert Panel on the Care of Older Adults with Multimorbidity. Guiding principles for the care of older adults with multimorbidity: an approach for clinicians. J Am Geriatr Soc 2012;60:E1-25.

9. Calderón-Larrañaga A, Poblador-Plou B, González-Rubio F, et al. Multimorbidity, polypharmacy, referrals, and adverse drug events: are we doing things well? Br J Gen Pract 2012;62:e821-6.

10. Koper D, Kamenski G, Flamm M, et al. Frequency of medication errors in primary care patients with polypharmacy. Fam Pract 2013;30:313-19.

11. McCann L, Lloyd F, Parsons $\mathrm{C}$, et al. 'They come with multiple morbidities': a qualitative assessment of pharmacist prescribing. $J$ Interprof Care 2012;26:127-33.

12. Kohn LT, Corrigan JM, Donaldson MS, eds. Committee on Quality of Health Care in America, Institute of Medicine. Washington, DC, USA: National Academies Press; 2000.

13. Kuzel AJ, Woolf SH, Engel JD, et al. Making the case for a qualitative study of medical errors in primary care. Qual Health Res 2003;13:743-80.

14. Jerak-Zuiderant S. Certain uncertainties: modes of patient safety in healthcare. Soc Stud Sci 2012;42:732-52.

15. Hor S, Godbold N, Collier A, et al. Finding the patient in patient safety. Health 2013;17:567-83.

16. Dixon-Woods M. Essay: why is patient safety so hard? A selective review of ethnographic studies. J Health Serv Res Pol 2010;15 (Suppl 1):11-16.

17. McDonald R, Waring J, Harrison S, et al. An ethnographic study of threats to patient safety in the operating theatre. Final report of a study funded by the Patient Safety Research Programme. http:// www.birmingham.ac.uk/Documents/college-mds/haps/projects/cfhep/ psrp/finalreports/PS008FinalReportHarrison.pdf (accessed 1 Oct 2013).

18. Healey A, Vincent C. Enhancing safety in surgery. Report of a series of studies funded by the National Patient Safety Research Programme. http://www.birmingham.ac.uk/Documents/college-mds/ haps/projects/cfhep/psrp/finalreports/PS009FinalReportVincent.pdf (accessed 1 Oct 2013)

19. Catchpole K, Gooden PJ, Giddings AEB, et al. Identifying and reducing errors in the operating theatre. Final report to the Patient Safety Research Programme. http://www.birmingham.ac.uk/ Documents/college-mds/haps/projects/cfhep/psrp/finalreports/ PS012FinalReportDeLeval.pdf (accessed 1 Oct 2013).

20. Woloshynowych M, Davis R, Brown R, et al. Enhancing safety in accident and emergency care. Report of a series of studies funded by the National Patient Safety Research Programme. http://www. birmingham.ac.uk/Documents/college-mds/haps/projects/cfhep/psrp/ finalreports/PS010AandEVincentetal2007.pdf (accessed 1 Oct 2013) 
21. Andrews LB, Stocking C, Krizek T, et al. An alternative strategy for studying adverse events in medical care. Lancet 1997;349:308-13.

22. Taxis K, Barber N. Ethnographic study of the incidence and severity of intravenous drug errors. BMJ 2003;326:1-4.

23. Kuzel AJ, Woolf SH, Gilchrist VJ, et al. Patient reports of preventable problems and harms in primary health care. Ann Fam Med 2004;2:333-40.

24. Burgess C, Cowie L, Gulliford M. Patients' perceptions of error in long-term illness care: qualitative study. J Health Serv Res Pol 2012;17:181-7

25. Buetow S, Kiata L, Liew T, et al. Approaches to reducing the most important patient errors in primary health-care: patient and professional perspectives. Health Soc Care Comm 2010;18:296-303.

26. Mason B, Epiphaniou E, Nanton V, et al. Coordination of care for individuals with advanced progressive conditions: a multi-site ethnographic and serial interview study. Br J Gen Pract 2013;63:e580-8.

27. Campbell R, Pound P, Morgan M, et al. Evaluating meta-ethnography: systematic analysis and synthesis of qualitative research. Health Technol Assess 2011;15:1-164.

28. Ford J. Improving quality of care in older adults with multi-morbidity: the need for a shift towards individualised patient-centred goals [Editorial]. J Gen Pract 2013;1:2. http://dx.doi.org/10.4172/2329$9126.1000 \mathrm{e} 103$.

29. Davis RE, Jacklin R, Sevdalis N, et al. Patient involvement in patient safety: what factors influence patient participation and engagement? Health Expect 2013;10:259-67.

30. O'Brien R, Wyke S, Guthrie B, et al. An 'endless struggle': a qualitative study of general practitioners' and practice nurses' experiences of managing multimorbidity in socio-economically deprived areas of Scotland. Chron IIIn 2010;7:45-59.

31. Davis RE, Sevdalis N, Jacklin R, et al. An examination of opportunities for the active patient in improving patient safety [Review]. J Pat Saf 2012;8:36-43.

32. Boote J, Barber R, Cooper C. Principles and indicators of successful consumer involvement in NHS research: results of a Delphi study and subgroup analysis. Health Pol 2006;75:280-97.

33. Agar $\mathrm{MH}$. The professional stranger. An informal introduction to ethnography. 2nd edn. San Diego, CA: Academic Press, 1996.

34. Hermanowicz JC. The longitudinal qualitative interview. Qual Sociol 2013;36:189-208.

35. Calman L, Brunton L, Molassiotis A. Developing longitudinal qualitative designs: lessons learned and recommendations for health services research [Correspondence]. BMC Med Res Method 2013;13:14. http://www.biomedcentral.com/1471-2288/13/14

36. Murray SA, Kendall M, Carduff E, et al. Use of serial qualitative interviews to understand patients' evolving experiences and needs. BMJ 2009;339:b3702.

37. Pinnock $\mathrm{H}$, Kendall M, Murray SA, et al. Living and dying with sever chronic obstructive pulmonary disease: multi-perspective longitudinal qualitative study. BMJ 2011;342:d142.

38. Harrison T, Taylor J, Fredland N, et al. A qualitative analysis of life course adjustment to multiple morbidity and disability. Res Gerontol Nurs 2013:6:57-69.

39. Public Health England Longer Lives 2013 [web site]. http:// longerlives.phe.org.uk/area-details\#are/E08000003/par/E9200000 (accessed 12 Jun 2013) 